Revue de droit comparé du travail et de la sécurité sociale

1 | 2018

Doctrine

\title{
Le droit social dans une société post-industrielle
}

\section{Anna Aleksandrova}

\section{(2) OpenEdition}

Journals

Édition électronique

URL : https://journals.openedition.org/rdctss/2001

DOI : 10.4000/rdctss.2001

ISSN : 2262-9815

Éditeur

Centre de droit comparé du travail et de la sécurité sociale

Édition imprimée

Date de publication : 1 avril 2018

Pagination : $90-101$

ISSN : 2117-4350

\section{Référence électronique}

Anna Aleksandrova, "Le droit social dans une société post-industrielle », Revue de droit comparé du travail et de la sécurité sociale [En ligne], 1 | 2018, mis en ligne le 01 novembre 2021, consulté le 12 novembre 2021. URL : http://journals.openedition.org/rdctss/2001 ; DOI : https://doi.org/10.4000/ rdctss.2001 Licence Creative Commons Attribution - Pas d'Utilisation Commerciale - Pas de Modification 4.0 International. 


\section{LE DROIT SOCIAL DANS UNE SOCIÉTÉ POST-INDUSTRIELLE}

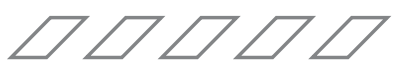

\section{RÉSUMÉ}

Cette contribution cherche à analyser les principales caractéristiques du droit social dans les conditions d'une société post-industrielle et à relever les tendances générales du droit social moderne, telles que l'individualisation, l'internationalisation, l'expansion de la contractualisation, l'accent mis sur le ciblage, la structuration selon les différentes phases du parcours de vie, etc.

MOTS CLÉS : Post-industrialisme, Droit social, personne, État, individualisation.

\section{ABSTRACT}

The paper deals with the concept and main features of social law, which is formed in the conditions of a postindustrial society. The main tendencies of development of modern social law, such as individualization, internationalization, expansion of contractual regulation, emphasis on targeting, adequacy, structuring at various stages of life, etc. are considered.

KEYWORDS : Post-Industrialism, Social Law, Person, State, Individualization. 


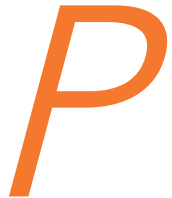

lusieurs chercheurs considèrent que la société contemporaine connait"une révolution" - post-industrielle, informatique, numérique, etc. ${ }^{-1}$, et mettent en exergue l'importance et le caractère fondamental des changements sociétaux ces dernières décennies. Ces changements sont conditionnés par la transformation du système économique : la prédominance du secteur des services sur le secteur de la production industrielle, l'auto matisation et l'informatisation des processus de production, l'évolution de la nature du travail (la prédominance du travail intellectuel et créatif sur le travail manuel). De plus, au cours des dernières décennies, «la croissance de la richesse est une grandeur variable, indépendante de la croissance de la production industrielle ${ }^{2}$. Le facteur clé du développement économique est le capital humain, le potentiel créatif de la personne.

À cet égard, on peut constater l'accroissement du rôle du droit qui assure "l'investissement » public dans le capital humain, règle les relations de travail, la santé publique, la sécurité sociale, etc. Les normes juridiques applicables sur ces sujets relèvent du domaine du droit social .

Le but général de notre contribution est de révéler et analyser les principales tendances de l'évolution du droit social durant la transition de la société vers le post-industrialisme. Pour ce qui nous concerne, le début de la transition vers une société post-industrielle se situe dans les années 1970. II n'existe pas de consensus sur ce point dans la doctrine; nous prenons comme référence le point de vue de l'un des fondateurs du concept de société post-industrielle, le sociologue américain Daniel Bell³.

Notre étude est basée sur les principes méthodologiques formulés par de grands théoriciens du droit russe, tels que S.S. Alekseev, A.B. Vengerov, M.N. Marchenko, etc. En particulier, nous utilisons la notion de tendances du développement du droit, proposée par le professeur M.N. Marchenko selon lequel « ce sont des directions émergentes du développement du droit, témoignant d'une évolution de la matière juridique en général (au niveau mondial ou autre), de ses branches ou de ses institutions $»^{4}$. Parmi les tendances du développement du droit, M.N. Marchenko distingue les tendances locales, régionales et mondiales; des tendances qui se forment et celles déjà formées ; tendances systèmeformant et tendances système-destructeur etc. ${ }^{5}$

1 Voir: Галгано Ф. "Объявленная революция" in Известия вузов. Правоведение. 2013, nº 3, p. 12.

2 Ibidem.

3 D. Bell, The Coming of Post-Industrial Society: A venture of social forecasting, New York Basic Books, 1973, 507p.

4 Марченко М.Н. Тенденции развития права в современно мире: учебное пособие. М.: Проспект, 2015, р. 93.

5 lbid., p.5. 


\section{I - QU’EST-CE QU'UNE « SOCIÉTÉ POST-INDUSTRIELLE »?}

A la charnière des XXème et XXlème siècles, des changements fondamentaux, caractérisés par le terme "révolution » se sont produits dans nos sociétés. II s'agit, en particulier, de la révolution post-industrielle, numérique, multimédia, managériale, globale, etc. ${ }^{6}$ Compte tenu de I'utilisation universelle d'Internet et de la transmission numérique de l'information, des chercheurs parlent de quatrième révolution industrielle (la transition vers une économie numérique $)^{7}$.

La révolution numérique affecte «non seulement le mode de production mais aussi le mode de commerce et de consommation, ou, en termes simples, de communication - et pousse les relations humaines « en dehors du temps et de l'espace, dans un espace non physique, le cyberespace $»^{8}$. A cet égard, on utilise aussi les termes de "révolution spatiale ${ }^{9}$ ou de " révolution multimédia ${ }^{10}$. Les produits de l'information et des communications jouent un rôle important dans l'économie moderne. F. Galgano a fait remarquer que «le mot "produit » est dématérialisé, il est utilisé pour signifier des « produits financiers ». Il faut aussi souligner que la richesse doit être dématérialisée afin de pouvoir être internationalisée ${ }^{11}$ (virements de compte à compte, cartes bancaires, argent numérique, etc.).

On peut considérer la révolution numérique comme une transition vers une société électronique, une société d'informatique dans laquelle une machine contrôlée par un ordinateur remplace le travail humain. L'automatisation de la production entraîne évidemment une réduction du nombre de travailleurs dans l'industrie. Des chercheurs parlent de révolution managériale ou néo-managériale ${ }^{12}$, et attirent l'attention sur l'évolution de la structure sociale de la société (augmentation du nombre de «cols blancs » par rapport aux travailleurs).

Le terme "révolution post-industrielle " a été utilisé pour la première fois par le sociologue Daniel Bell ${ }^{13}$ pour décrire le processus de transition vers un nouveau type de société fondée sur la connaissance (science). En 1959, lors d'un séminaire international à Salzbourg, D. Bell a inventé la notion de "société post-industrielle " dans le sens qui est maintenant largement reconnu - pour désigner une société où le secteur industriel est en train de perdre son rôle dominant (en raison d'une " technologisation » croissante),

6 Галгано Ф. "Объявленная революция" іn Известия вузов. Правоведение. 2013, n 3, p. 12.

7 Чесалина О.В. "Работа на основе интернет-платформ (crowdwork и work on demand via apps) как вызов трудовому и социальному праву" in Трудовое право в России и за рубежом. 2017, $\mathrm{n}^{\circ} 1$, p. 52-55.

8 Галгано Ф. Op.cit., p. 13.

9 S. Ortino, Il nuovo Nomos della Terra, Bologna, 2000.

10 A.Baldassar. Globalizzatione contro democrazia. Roma, Bari, 2002.

11 Галгано Ф. Op.cit., p.12.

12 A.D. Chandler, Dimensione e diversificazione. Le dinamiche del capitalismo industriale, Bologna, 1994.

13 D. Bell. The Coming of Post-Industrial Society: A venture of social forecasting. New York: Basic Books, 1973. $507 \mathrm{p}$. 
la science devenant une force principale productive. Le potentiel de développement de cette société est de plus en plus déterminé par le niveau d'information et des connaissances ${ }^{14}$.

D. Bell opposait le concept de "post-industriel » aux concepts de " pré-industriel » et « industriel ». A son avis, le secteur pré-industriel est principalement basé sur l'agriculture, l'exploitation minière, la pêche, le stockage du bois et d'autres ressources (y compris le gaz naturel ou le pétrole). Le secteur industriel est toujours producteur, il utilise l'énergie et la technologie des machines pour la fabrication des biens. Le secteur post-industriel est de transformation, «ici l'échange d'informations et de connaissances se produit principalement au moyen des télécommunications et des ordinateurs ${ }^{15}$.

Les caractéristiques essentielles de la société postindustrielle, selon le concept de D. Bell, sont : la prédominance de la sphère des services sur la production (" une société basée sur les services »), l'émergence de nouveaux services (" services humanitaires - éducation, santé, et services professionnels - analyse et planification, design, programmation, etc. «), le rôle croissant des connaissances théoriques dans la production de biens et de services et, par conséquent, l'importance de l'éducation ${ }^{16}$.

En outre, ce nouveau type de société se caractérise par un changement dans la composition de la main-d'œuvre (diminution du nombre de travailleurs et augmentation du nombre de "cols blancs ${ }^{17}$ ) et une modification de la structure sociale de la société ( " dans une société post-industrielle, le statut d'une personne dépend de son niveau d'éducation ${ }^{18}$ ).

On a tendance à penser que la formation d'une société post-industrielle aurait eu lieu du début des années 1970 jusqu'à la fin des années 1980, alors que certaines tendances (comme la domination de la sphère des services sur la production matérielle) sont apparues juste après la seconde guerre mondiale.

L'idée de considérer la société émergente comme une société post-industrielle a été reprise par des représentants de diverses écoles scientifiques. Les études de K. Tominaga, R. Dahrendorf, H. Kahn confirment le caractère fondamental et radical des modifications de la société contemporaine. La plupart des chercheurs considère que l'accroissement du rôle des sciences et des changements technologiques est une des bases de ces changements. Aujourd'hui, les chercheurs soulignent que "la personne clé dans la société post-industrielle est la personne créative ${ }^{19}$, en d'autres termes, c'est le capital humain qui devient le facteur déterminant du développement de l'économie.

14 Иноземцев В.^. Современное постиндустриальное общество: природа, противоречия, перспективы. М.: Аогос, 2000. http://filosof.historic.ru/books/item/f00/s00/z0000946/st003. shtml

15 Бемл А. Грядущее постиндустриальное общество. Опыт социального прогнозирования. Пер. с англ. В.А. Иноземцева. М. : Academia, 1999, р. 158.

16 Бем^ А. Грядущее постиндустриальное общество: опыт социального прогнозирования. ИзА. 2-ое, испр. и Аоп. Пер. с англ. В.А. Иноземцева. М.: Academia, 2004, р. 73.

17 Галгано Ф. Op.cit., р. 14.

18 Бем^ А. Грядущее постиндустриальное общество: опыт социального прогнозирования. ИзА. 2-ое, испр. и Аоп., р. 63.

19 Ерохин С.Г. Человеческий капитал в постиндустриальном обществе. М.: Издательскоторговая корпорация «Аашков и К», 2012, р. 4. 
Il est évident que dans la société de " high tech", c'est le potentiel créateur de la personne humaine qui acquiert la plus grande valeur, il est un « instrument du travail » grâce auquel les biens publics sont créés ${ }^{20}$. Le rôle principal sur le marché mondial appartient aux États qui peuvent utiliser non seulement des ressources naturelles mais aussi le capital humain, les inventions et les idées scientifiques. À cet égard, la question des moyens juridiques pour la formation et l'amélioration du capital humain est d'une importance particulière.

Dans les travaux de recherche en sciences économiques, on souligne que «la société doit avoir un potentiel mental et physique, qui est la base du capital humain, lequel à son tour, peut être formé en raison du développement effectif de la sphère sociale $»^{21}$. Par conséquent, en société post-industrielle, les priorités en termes d'activité économique dans la sphère sociale seront : la santé, l'éducation et la culture, ainsi que le logement et les services collectifs ${ }^{22}$.

Dans la société post-industrielle, comme indiqué ci-dessus, la créativité humaine est de plus en plus importante, si bien qu'aujourd'hui, les capacités intellectuelles et sociales de la personne deviennent « la base de la formation et de l'amélioration des capacités économiques ${ }^{23}$. Cependant, la position du gouvernement russe en ce qui concerne la formation du capital humain est principalement technocratique. On doit constater une attention insuffisante des pouvoirs publics aux problèmes d'éducation, de science fondamentale, de sécurité sociale ainsi qu'un fonctionnement inefficace du système de fabrication des produits de haute technologie ${ }^{24}$.

Les experts dans le domaine du droit du travail expriment des opinions similaires. En 2005, le Professeur. A.M.Kurennoy écrivait : " Il faut rappeler, non pour la première fois, une vérité presque banale, que toute économie (particulièrement, l'économie de marché) est impossible sans utilisation de la main-d'œuvre humaine ce qu'oublient les hommes politiques russes, les économistes, les employeurs, et parfois les juristes. Ils mettent au premier plan les questions de propriété, de statut juridique des entreprises, d'investissement, de bénéfices, d'impôts. (...) Ce manque de compréhension ouvre la voie à des conflits sociaux $»{ }^{25}$

\section{II - LA NOTION DE « DROIT SOCIAL POST-INDUSTRIEL »}

Au cours des dernières décennies, de nombreux ouvrages scientifiques ont été consacrés à la société post-industrielle. Cependant, le droit post-industriel est devenu un objet de recherche il n'y a pas longtemps. On peut trouver des éléments du concept de

20 Ibid.p. 10

21 Яковлев А.С. Роль социальной сферы в формировании постиндустриального общества в России. Орел: ОрелГАУ, 2012, р. 10.

22 Ibid., p. 12.

23 Ерохин С.Г. Ор. cit., р. 10.

$24 \mathrm{lbid}$, p.5.

25 Куренной А.М. "Новое законодательство о труде: итог или этап?" in Ученые-юристы МГУ о современном праве. Под реА. М.К. Треушникова. М.: ОАО « Издательский дом « Городец », 2005, p. 96. 
droit post-industriel dans les travaux de F. Galgano ${ }^{26}$, I.L. Chestnov ${ }^{27}$, M.N. Marchenko ${ }^{28}$, G. Valleé29, M.-A. Moreau ${ }^{30}$ entre autres.II n'est pas ici possible d'analyser en détail tous les concepts scientifiques concernant le droit post-industriel. Nous nous bornerons donc à esquisser notre point de vue sur la notion et les caractéristiques principales du droit postindustriel.

Le droit post-industriel est un droit qui se forme à un nouveau stade du développement social (à l'époque post-industrielle, depuis 1970), qui exprime les tendances générales de l'évolution de la société post-fordiste et qui est un moyen de la politique publique, un instrument d'intervention de l'Etat sur les rapports sociaux afin de surmonter les conséquences négatives de la mondialisation et de l'informatisation.

Les caractéristiques principales du droit post-industriel sont:

- la nature multi-dimentionnelle - pluralité des sujets créateurs de règles de droit (I'Etat et d'autres sujets) ; on parle aussi de « privatisation » du droit ;

- la nature globale (exterritorialité) ;

- I'internationalisation (augmentation du rôle de la réglementation juridique internationale);

- la dimension anthropologique (individualisation, redoublement de l'attention sur l'homme comme personnalité);

- la contractualisation (expansion des principes contractuels), notamment dans le domaine du droit public ;

- l'augmentation du rôle du juge dans le processus de création et de mise en œuvre des règles de droit.

Quant au droit social post-industriel, il faut mentionner qu'il s'agit d'un élément inaliénable et, à notre avis, de l'élément le plus important du système de droit postindustriel (il présente par voie de conséquence les caractéristiques fondamentales du droit post-industriel dans son ensemble).

Chaque étude en droit social actuel acquiert le caractère interdisciplinaire parce que les questions de protection sociale sont indissolublement liées à la sphère économique (ressources de l'Etat, niveau de vie de la population, etc.) et sociologique (concept et caractéristiques de la société post-industrielle, particularités du développement social, processus de formation de la personne humaine, etc.). En même temps, en étudiant le droit social on ne peut pas se passer de catégories purement juridiques. En particulier, il est nécessaire de déterminer ce qu'est le droit social.

26 Галгано Ф. Op.cit., p. 11-33.

27 Честнов И.^. "Постклассическое правопонимание" іn Общественные науки и современность. 2010, n 5, p. 157-162 ; Честнов И.^. "Что есть право?" іп Известия вузов. Правоведение. 2013, $n^{\circ} 3$, p. 229-237.

28 Марченко М.H. op.cit.

29 G. Vallée, "Diversité des modes d'action de l'État dans le domaine social et le système de relations industrielles" in L'État à l'épreuve du social. Paris, 1998.

30 M.A. Moreau, Normes sociales, droit du travail et mondialisation, Paris, Dalloz, 2006. 
Ces dernières années, les chercheurs russes utilisent largement la notion de « droit social ", bien qu'il n'y ait pas de consensus sur son contenu. En particulier, N.V. Putilo considère le droit social comme un ensemble de normes qui règlent des rapports pour garantir à tous un accès aux biens élémentaires (éducation, santé, logement, sécurité sociale, etc.) - c'est-à-dire, une sphère plus large que l'objet du droit du travail et du droit de la sécurité sociale. ${ }^{31}$

V.A. Uvachev estime que "le droit social comprend les droits de I'homme socioéconomiques et culturels, qu'il a son objet, sa méthode et ses principes unifiés de régulation $»^{32}$. La plupart des chercheurs russes (P.G. Glushchenko, G.A. Korobov, A.I. Mazilov, E.N. Milyukova, S.D. Solovyov) considèrent que la protection sociale fait partie du droit social. Ainsi, le droit social post-industriel comprend les normes qui régissent les relations dans le domaine du travail et de la sécurité sociale. Une approche similaire est retenue dans les travaux des chercheurs européens ${ }^{33}$.

\section{III - TENDANCES DU DROIT SOCIAL POST-INDUSTRIEL}

Quelles sont les tendances de l'évolution du droit du travail et de la sécurité sociale à l'époque post-industrielle? Tout d'abord, il faut relever les traits inhérents au droit postindustriel dans son ensemble. Premièrement, c'est une individualisation. Elle s'exprime par une attention accrue du législateur vis-à-vis du travailleur comme personne, comme individualité porteur de certaines qualités, de croyances et de caractéristiques. Ainsi, ont été consacrées par la loi les libertés d'opinion, d'expression, d'idéologie et de religion sur le lieu de travail ; les droits des minorités ; la protection des données personnelles du salarié ; la prohibition des discriminations (genre, âge, religion, convictions, etc.) On peut donner en exemple, la loi sur l'égalité de traitement dans les relations de travail (Allemagne) ${ }^{34}$, la loi contre les discriminations (France) ${ }^{35}$, la loi sur l'équité en matière d'emploi (GrandeBretagne $)^{36}$, etc. Le Code du travail de la Russie, adopté en $2001^{37}$, contient un chapitre 14, consacré à la protection des données personnelles du salarié. Les articles 2 et 3 du Code proclament l'interdiction des discriminations au travail, principe par ailleurs concrétisé dans de nombreuses dispositions du Code (parties 2-6).

31 Путило Н.В.Социальные права граждан: история и современность. М.: ИА «Юриспруденция», 2007, p.7.

32 Увачев В.А. Социально-правовое государство и гражданское общество постиндустриальной эпохи: правовые основы функционирования и взаимодействия (на примере стран Западной Европы и США). Автореф. Аисс. к.ю.н. М.: Российский государственный социальный университет, 2006, р. 14.

33 A. Supiot (dir.), "Au-delà de l'emploi. Transformations du travail et devenir du droit du travail en Europe (Extraits du rapport)", Le Droit Ouvrier. 2015, n 807, p. 568.

34 Allgemeines Gleichbehandlungsgesetz.14 August 2006, Bundesgesetzblatt. I 2006. S. 1897.

35 Loi n ${ }^{\circ}$ 2008-496 du 27 mai 2008 portant diverses dispositions d'adaptation au droit communautaire dans le domaine de la lutte contre les discriminations, Journal Officiel, 28 mai 2008.

36 The Employment Equality (Age) Regulations. 3 avril 2006, http://www.opsi.gov.uk/si/ si2006/20061031.htm

37 Трудовой кодекс Российской Федерации от 30 декабря 2001 г. N 197-Ф3 (ред. от 31 декабря 2017), http://www.consultant.ru/document/cons doc LAW 34683/ 
Dans une société post-industrielle, la nature du travail change : le travail physique est de plus en plus remplacé par le travailintellectuel. À cet égard, le taux de risques psychosociaux auxquels une personne est exposée sur le lieu de travail augmente. Le droit social devient donc de plus en plus préventif, c'est-à-dire qu'il vise moins à restaurer (santé, statut financier, statut social d'un travailleur) qu'à prévenir les risques sociaux.

Une autre tendance est l'internationalisation du droit social, qui se traduit par l'expansion de pratiques d'emprunt de normes en vigueur dans d'autres pays et par l'accroissement du rôle de la réglementation internationale. Parmi les textes qui ont influencé le développement du droit social national, on peut mentionner le Pacte international relatif aux droits économiques, sociaux et culturels de 1966, la Charte sociale européenne (rédactions de 1961 et 1996), ou le Code européen de la sécurité sociale de 1964.

L'élaboration de standards internationaux dans le domaine de la protection sociale acquiert une importance particulière. Le rôle principal dans ce processus revient à I'Organisation internationale du travail (OIT ci-après). L'OIT a adopté un grand nombre de conventions dont la Convention $n^{\circ} 102$ concernant la sécurité sociale (norme minimum), la Convention $n^{\circ} 118$ sur l'égalité de traitement (sécurité sociale), la Convention $n^{\circ} 128$ concernant les prestations d'invalidité, de vieillesse et de survivants, la Convention $n^{\circ} 157$ sur la conservation des droits en matière de sécurité sociale. Les garanties les plus importantes pour la protection de l'enfance et de la maternité sont établies respectivement par la Convention $n^{\circ} 182$ sur les pires formes de travail des enfants et par la Convention $n^{\circ} 183$ sur la protection de la maternité. Malheureusement, de nombreux pays n'ont pas ratifié les Conventions $n^{\circ} 128$ et $n^{\circ} 157$ de l'OIT. La Russie travaille actuellement à aligner sa législation sur les exigences de la Convention nº 102 de l'OIT qui, avec les deux conventions susmentionnées, n'a pas encore été ratifiée par notre pays.

Une autre tendance du droit social moderne réside dans l'unification progressive de ses normes. Actuellement, les frontières entre les différents modèles d'Etat social s'écroulent ${ }^{38}$. La législation sociale s'unifie successivement. On peut trouver des exemples dans la législation sur les retraites avec l'introduction universelle d'éléments de capitalisation dans les régimes de retraite, l'accroissement de la participation des fonds privés au système de l'épargne-retraite, ou encore l'élévation de l'âge de la retraite, etc. Bien sûr, l'unification est le résultat de l'introduction de standards internationaux dans le domaine de la protection sociale, ce qui est sans doute positif.

Au début des années 2000, les chercheurs en droit social soulignaient déjà de telles tendances telles que l'expansion des principes contractuels ${ }^{39}$ ou le rôle croissant du juge dans le processus de mise en œuvre des normes juridiques ${ }^{40}$. En particulier, dans le domaine social, le contrat peut prendre diverses formes: le contrat comme moyen de régulation des

38 Voir Сидорина Т.Ю. "Институты самоорганизации граждан и развитие теории государства всеобщего благосостояния" in Общественные науки и современность. 2010, №5, p. 93.

$39 \mathrm{Ph}$. Auvergnon (dir.), La contractualisation du droit social. Actes du séminaire international de droit comparé du travail, des relations professionnelles et de la sécurité sociale, Bordeaux, Centre de droit comparé du travail et de la sécurité sociale, 2003.

40 Ph. Auvergnon (dir.), Les juges et le droit social : contributions à une approche comparative. Actes du séminaire international de droit comparé du travail, des relations professionnelles et de la sécurité social, Bordeaux, Centre de droit comparé du travail et de la sécurité sociale, 2002. 
relations entre les différents acteurs de la protection sociale (organismes publics et privés, services médicaux et sociaux, représentants de certaines professions -médecins, avocats) ; le contrat comme un moyen d'influencer les personnes bénéficiant de l'aide sociale (conclu entre les bénéficiaires d'une part et les services sociaux et médicaux de l'Etat d'autre part) ${ }^{41}$.

En Russie, le concept de "contrat social » est actuellement développé par A.M. Luchnikov et M.V. Luchnikova ${ }^{42}$. Ils en relèvent les caractéristiques suivantes :

- la sphère de régulation : le contrat est conclu pour établir, changer ou protéger les droits sociaux);

- la nature personnelle : les droits et obligations découlant d'un « contrat social » ne peuvent, en règle générale, pas être transférés à une autre personne);

- les contrats sociaux ne sont régis que par les normes de la législation sociale ;

- la volonté des parties au contrat social est très limitée ;

- les contrats sociaux sont basés sur l'unité des principes privés et publics ;

la procédure de conclusion, de modification et de résiliation des contrats sociaux est réglementée en détail par la législation (il est permis de conclure uniquement les contrats directement prévus par la législation sociale).

La tendance à la « privatisation » du droit social a trouvé son expression sous la forme de la responsabilité sociale des entreprises (RSE). La Commission des Communautés européennes définissait la RSE comme "l'intégration volontaire par les entreprises de préoccupations sociales et environnementales à leurs activités commerciales et leurs relations avec leurs parties prenantes $»^{43}$ car elles sont de plus en plus conscientes qu'un comportement responsable se traduit par une réussite commerciale durable. La RSE a une nature volontaire, mais les autorités européennes et nationales sont intervenues pour promouvoir sa pratique et stimuler son développement. Ces dernières années, la RSE a fait l'objet de propositions d'institutionnalisation et de légalisation. ${ }^{44}$.

Plus généralement, on voudrait mettre en exergue une incohérence paradoxale du droit social post-industriel. D'une part, il se caractérise par la tendance à l'humanisation: élargissement des garanties aux catégories de travailleurs socialement faibles (personnes ayant des responsabilités familiales, handicapés, jeunes, etc.), inclusion dans le droit du travail de nouvelles formes de travail (domestiques, télétravailleurs, etc.), création de possibilités d'épanouissement personnel sur le lieu de travail (concept de travail décent), conditions pour restaurer la santé et le bien-être du travailleur. Les exemples ne manquent pas. En Europe, ces dernières années, un certain nombre de lois ont été adoptées en vue d'assurer la combinaison optimale entre vie professionnelle et vie familiale : la loi sur le travail

41 R. Lafore R., « Le contrat dans la protection sociale, une approche française » in La contractualisation du droit social : actes du séminaire international de droit comparé du travail, des relations professionnelles et de la sécurité sociale. Bordeaux, 2003, p. 203

42 Тарусина Н.Н., Аушников А.М., Аушникова М.В. Социальные договоры в праве. М.: Проспект, 2016.

43 Commission des Communautés européennes. Communication de la Commission concernant la Responsabilité sociale de l'entreprise : Une contribution des entreprises au developpement durable. Bruxelles, 2002, p. 3.

44 Чубарова Т. "Социальная ответственность работодателей в контексте мировой экономики" in Человек и труд. 2013, № 8, p. 56-57. 
et les soins (Pays-Bas)45, la loi sur les soins (Allemagne) ${ }^{46}$, la loi sur les congés parentaux (Royaume-Uni) ${ }^{47}$, le décret-loi sur la conciliation entre soin, vie et travail (Italie) ${ }^{48}$. La Russie avait déjà une législation développée dans ce domaine (héritée de l'Union Soviétique). En particulier, à la fin du XXe siècle, le congé maternité en Russie (rémunéré à $100 \%$ du salaire moyen) était de 140 jours. La mère ou le père de l'enfant (ou une autre personne pouvait prendre soin d'un enfant jusqu'à 1,5 ans), pouvait avoir un congé parental payé à $40 \%$ du salaire moyen et travailler à temps partiel, si elle/il le voulait. Ces garanties sont toujours en vigueur en droit russe.

D'autre part, la tendance à la déshumanisation du droit social est tout aussi évidente : bon nombre des obligations financières dans le domaine de la protection sociale (retraite, assurance maladie, etc) ont été transférées de l'Etat aux institutions privées, à l'employeur, voire au travailleur lui-même ; le cercle des retraités est réduit (grâce à l'élévation de l'âge de la retraite, à la réduction des possibilités de retraite anticipée, etc.) ; la «flexibilité » a été renforcée. Actuellement, l'âge normal de la retraite est de 65 ans; les réformes législatives récentes (en Allemagne, Grèce, aux Etats-Unis, etc.) prévoient de le porter à 67 ans et plus. En Russie, l'âge de la retraite est aujourd'hui l'un des plus bas au monde (55 ans pour les femmes et 60 ans pour les hommes) - cela peut être considéré comme une survivance de l'ère socialiste. Cependant, en 2016 on a adopté la loi49 prévoyant l'augmentation progressive de l'âge de la retraite pour les fonctionnaires jusqu'à 65 ans pour les hommes et 63 ans pour les femmes. En ce qui concerne le reste de la population, l'augmentation de l'âge de la retraite n'est possible que lorsqu'il y a allongement de la durée de vie, surtout pour les hommes (en 2016, la durée de vie en Russie était de 66,5 ans pour les hommes et 77 ans pour les femmes ${ }^{50}$ ).

L'Association internationale de la sécurité sociale (AISS ci-après) dans son rapport de 2016 a déterminé les tendances du développement des systèmes de sécurité sociale en Europe $^{51}$, par les éléments suivants :

- la volonté d'élever l'adéquation des prestations au rang de priorité ;

- le recours, dans le cadre d'un système universel, à un ciblage intelligent pour répondre aux besoins spécifiques des populations vulnérables ;

- une intervention de la protection sociale structurée selon les différentes phases du parcours de vie ;

45 Wet Arbeid en Zorg, Stb 2001/567 // https://zoek.officielebekendmakingen.nl/stb-2001-567.html

46 Pflege-Weiterentwicklungsgesetz. 28.05.2008. URL: http://www.buzer.de/gesetz/8223/index.htm

47 The Shared Parental Leave Regulations 2014. URL:

https://www.legislation.gov.uk/ukdsi/2014/9780111118856/regulation/6

48 Decreto Legislativo 15 giugno 2015, n. 80. URL: http://www.gazzettaufficiale.it/eli/id/2015/06/24/15G00094/sg

49 Федеральный закон от 23 мая 2016 г. N 143-Ф3 «О внесении изменений в отдельные законодательные акты Российской Федерации в части увеличения пенсионного возраста отАельным категориям граждан » // http://www.consultant.ru/document/cons doc LAW 198199/

50 https://ruxpert.ru/Статистика:Продолжительность жизни в России

51 Europe: approches stratégiques pour renforcer la sécurité sociale. Développements et tendances 2016. Geneve: Association internationale de la sécurité sociale (AISS), 2016, p. IV-VI. 
- I'introduction d'une certaine flexibilité dans la fourniture des prestations, tenant compte des différences qui existent au sein de la population en matière de revenu, de besoin de soins de santé, de situation familiale et de situation professionnelle.

Le rapport de l'AISS constate que " les besoins restent élevés, mais aussi que les ressources financières sont de plus en plus limitées ${ }^{52}$. Ainsi, l'orientation de la sécurité sociale sur les besoins individuels et sa structuration selon les différentes phases du parcours de vie sont conditionnées non seulement par la volonté d'une humanisation accrue des systèmes de protection sociale, mais aussi par la nécessité d'optimiser les dépenses dans un contexte de crise économique.

Le législateur russe a également décidé de renforcer le ciblage des prestations dans le contexte de la crise économique. En particulier, l'allocation mensuelle pour un enfant (jusqu'à l'âge de 16 ans) n'est versée qu'aux citoyens nécessiteux (à faible revenu) ; la même condition est prévue pour le versement de la compensation des frais parentaux pour la garde de l'enfant ( " jardin d'enfants », crèche, centre aéré ou structure d'accueil pour enfant). La nouvelle allocation familiale introduite depuis le $1^{\text {er }}$ janvier 2018 (par le Président de la Russie) est également ciblée et versée aux familles à faible revenu pour le premier ou le deuxième enfant jusqu'à l'âge de 1 an et demi ${ }^{53}$.

Si autrefois les principes d'universalité et d'uniformité des allocations, proclamés dans le rapport de Lord W. Beveridge au Parlement britannique en 1942 semblaient la panacée pour affronter tous les maux sociaux, il y a aujourd'hui un changement de repères : on " s'arme " des idées d'adéquation et de ciblage. Plus globalement, il faut souligner le changement de paradigme des activités de l'État dans son ensemble : au tournant des $X X^{e}$ et $X X l^{e}$ siècles il y a un rejet de l'idée de "l'État-providence » (Welfare State) et le passage à un modèle d'État social basé sur le travail (Workfare State) $)^{54}$.

Ainsi, nous pouvons parler d'un nouveau cycle d'individualisation et de libéralisation dans les relations entre l'État et l'individu. Dans le domaine social, une part importante de la responsabilité en matière de protection sociale a été transférée à la personne ellemême. Les exemples incluent l'expansion de la capitalisation dans les régimes de retraite, la limitation des garanties dans le domaine de l'assurance maladie et de l'assurance retraite, etc. En même temps, l'État conserve, bien sûr, la charge de la protection des personnes qui, en principe, ne peuvent subvenir à leurs besoins (orphelins, invalides depuis l'enfance, etc.).

L'affaiblissement des principes de collectivité et de solidarité dans le domaine social est principalement dû à des raisons socio-économiques objectives (crises économique et démographique, instabilité financière des systèmes de protection sociale, passage à une société postindustrielle). L'idéologie du consumérisme, cultivée dans le monde de l'économie globale, ne contribue pas au renforcement de la solidarité. L'orientation en faveur des intérêts individuels s'observe partout: dans le domaine de l'éducation (formation

52 lbid., p. V.

53 Федеральный закон от 28.12.2017 N 418-Ф3 »О ежемесячных вып^атах семьям, имеющим Аетей »// http://www.consultant.ru/document/cons doc LAW 286470/

54 Voir: Чиркин B.Е. "Общечеловеческие ценности и современное государство” in Государство и право. 2002, № 2 ; Чиркин В.Е. "Россия, Конституция, Аостойная жизнь: анализ взаимосвязей" in Государство и право. 2006, № 5. 
continue), du développement des carrières, des rapports personnels (familiaux) etc.

L'économie numérique, malgré le flou apparent des frontières (libre circulation de l'information, du capital, du travail), ne fait que renforcer l'isolement de l'individu et la segmentation de la société. La vidéosurveillance et les méthodes numériques d'identification de la personne peuvent conduire à un contrôle total sur le processus de travail et sur le travailleur lui-même.

Cependant, la pratique montre qu'un rejet total des idées de solidarité dans le domaine de la protection sociale est impossible. La crise financière peut dévaloriser le capital individuel de retraite. La spécialisation (individualisation, ciblage) des allocations ne peut être illimitée, sinon elle réduit à néant les principes fondamentaux de la politique sociale (faut-il soutenir la maternité et l'enfance en général ou seulement les pauvres mères et enfants)?

Le principe de répartition dans les systèmes de retraite reste prioritaire (le principe cumulatif est complémentaire). Depuis plusieurs années, on constate un chômage croissant et une réduction du coût de la main-d'œuvre, des difficultés de financement de l'assurance sociale, etc. C'est pourquoi, dans certains pays (Japon, États-Unis, Finlande), il est proposé de passer au modèle du "revenu individuel inconditionnel " qui suppose le versement régulier d'une somme à chaque membre de la société. Le revenu individuel inconditionnel doit être versé à chaque citoyen, quel que soit son niveau de revenu et sans condition de travail.

Les spécialistes en droit social prêtent attention à l'expérience des pays africains en matière d'assistance mutuelle. Aux instruments financiers et aux principes d'accumulation peuvent être opposés des liens sociaux horizontaux et des idées de la solidarité : " c'est la solidarité de ceux qui sont tour à tour créanciers et débiteurs qui constitue la garantie la plus sûre de l'égale liberté et sécurité de chacun $»^{55}$. Ainsi, il y a des raisons de croire que l'« individualisme » actuel sera remplacé par une nouvelle solidarité sociale selon des configurations pour l'instant inconnues.

55 A. Supiot, «Vers un nouveau statut social attaché à la personne du travailleur? Mise en perspective des réformes depuis 2013 », Le Droit Ouvrier, Oct. 2015, nº 807, p. 566.

ANNA ALEKSANDROVA

PhD, chargé de cours à la faculté de droit de l'Université d'Etat de Penza, Russie.

Thèmes de recherche : droit constitutionnel comparé et droit social comparé.

Publications :

A. Aleksandrova, "Социальные права в конституциях XXI в." (" Les droits sociaux dans les constitutions du XXI siècle") in Журнал зарубежного законоАательства и сравнительного правоведения. (Revue de législation étrangère et de droit comparé) 2017, n 1, p. 47-53.

A. Aleksandrova, "Постинаустриализм и труАовое законоАательство" ("Le postindustrialisme et la législation du travail") in Трудовое право в России и за рубежом. (Le droit du travail en Russie et à l'étranger) 2017, n³, p. 7-10. 